\title{
Non-linear optics for transducers: principles and materials*
}

\author{
H J W M Hoekstra, G J M Krijnen, A Driessen, P V Lambeck and Th J A Popma \\ Lightwave Device Group of the MESA Research Institute, Untverstiy of Twente, $P O$ Box 217, 7500 AE Enschede (Netherlands) \\ (Received February 7, 1992, accepted March 20, 1992)
}

\begin{abstract}
This paper concentrates on intensity-dependent refractive-index changes due to the third-order optical non-linearity Materials exhibiting such effects are good candidates for applications in all-optical devices The discussion will be on these materials, and characterization techniques and an overview will be given of proposed and realized all-optical devices Of the latter, the non-linear Y-junction and Mach-Zehnder interferometer will be treated in considerable detall
\end{abstract}

\section{Introduction}

Since ancient times, light has been used as a carrier of information In technical applications, however, it played no significant role until the recent invention of the laser and the introduction of the optical fibre Nowadays, the fibre is widely used in communication networks, allowing for extremely dense information transfer that is not affected by unwanted perturbations like (other) electromagnetic radiation

For the processing of information, light has been used since the 1950s in analog or coherent optical computers, utılızıng the intrinsic parallelısm of light and also the fact that a single lens may perform a Fourier transform of a two-dimensional field pattern Such coherent optical computers have been used for the processing of information from radar screens, and are still used for process control in factories, e $\mathrm{g}$, to filter out the smaller details of a product, or to measure the mesh-size of canvas or the thickness of a thin fast-moving thread Most information processing today is done by digital electronic computers, which have an impressive and still-growing potential

However, it is expected that the increasing complexity of electronic computers will sooner or later

*Invited paper presented at the 6th International Conference on Solid-State Sensors and Actuators (Transducers '91), San Francisco, CA, USA, June 24-28, 1991 lead to a frustration of this growth, mainly due to the sequential communication in an electronic computer and also to the limited switching time of a transistor and the unwanted interference of signals To overcome these problems, at least in part, (integrated) optics may come into play The major advantages of applications of optics, for both communication and processing, are the intrinsic parallelism and also the potentially fast switching times $(<1 \mathrm{ps})$ of all-optical devices By choosing the appropriate ( 1 e, linear) materials, optical signals can propagate closely packed (in time or space) without affecting one another For communication over long distances, the optical fibre has been shown to be able to transmit data at several Gbit/s over thousands of kilometres Here optical (temporal) solitons propagate undistorted due to a balance of the group velocity dispersion and the non-linearity, at least if the losses are sufficiently well compensated, e g, by Er-doped fibre amplifiers pumped by laser diodes For all-optical processing, however, there is still a need for materials having a strong third-order non-linear optical (NLO) effect, with short ( $\approx 1 \mathrm{ps)}$ turn-on and turn-off tımes

This paper concentrates mainly on third-order NLO materials In Section 2 a short introduction will be given to the field of NLO and its appl1cability Section 3 will be concerned with a short discussion on NLO materials and on a few characterization techniques In Section 4 an overview will be given of a number of well-known third-order 
NLO devices Here the merits of the NLO Y-junctıon and Mach-Zehnder interferometer will be discussed in more detail

\section{A short introduction to non-linear optics}

The response of matter to one or more optical or d c fields may be described macroscopically by the polarization, $\boldsymbol{P}(\boldsymbol{r}, t)$ This quantity is in general a very complicated non-linear function of the history of the electric- and magnetic-field distributions If the effect of magnetic fields may be neglected, and if there is no permanent polarization, the polarization in a uniform medium is given by the following convolution in time and space [1]

$$
\begin{aligned}
& \boldsymbol{P}(\boldsymbol{r}, t)= \epsilon_{0} \sum_{n=1} \int \chi^{(n)}\left(\boldsymbol{r}-\boldsymbol{r}_{1}, \quad, \boldsymbol{r}-\boldsymbol{r}_{n},\right. \\
&\left.t-t_{1}, \quad, t-t_{n}\right) \\
& \cdot \boldsymbol{E}\left(\boldsymbol{r}_{1}, t_{1}\right) \quad \boldsymbol{E}\left(\boldsymbol{r}_{n}, t_{n}\right) \mathrm{d} \boldsymbol{r}_{1} \quad \mathrm{~d} \boldsymbol{r}_{n} \mathrm{~d} t
\end{aligned}
$$

Here $\chi^{(n)}$, the $n$th order susceptibility, is a tensor of rank $3^{n+1}$

It is convenient for both experimental and theoretical purposes to work with the Fourier transforms, with respect to $t$ and $r$, of the electric fields and the polarization As eqn (1) contains products of the electric field, care should be taken in using the complex notation The Fourier transform of the electric field is defined as

$$
\begin{aligned}
& \boldsymbol{E}(\boldsymbol{k}, \omega) \equiv \int \mathrm{d} \boldsymbol{r} \mathrm{d} t \boldsymbol{E}(\boldsymbol{r}, t) \exp \{1(\boldsymbol{k} \cdot \boldsymbol{r}-\omega t)\} \\
&=(2 \pi)^{4} \sum_{j}\left\{\boldsymbol{E}_{\boldsymbol{k}_{\jmath} \omega_{j}} \delta\left(\boldsymbol{k}-\boldsymbol{k}_{\jmath}\right) \delta\left(\omega-\omega_{\jmath}\right)\right. \\
& \\
&\left.+\boldsymbol{E}_{\boldsymbol{k}_{j} \omega_{j}}^{*} \delta\left(\boldsymbol{k}+\boldsymbol{k}_{j}\right) \delta\left(\omega+\omega_{\jmath}\right)\right\} / 2
\end{aligned}
$$

where we have used for the second equality the fact that the total electric field, $E(r, t)$, of course being equal to the inverse transform of eqn (2a), can be expressed, in the presence of a number of plane waves, as

$$
\boldsymbol{E}(\boldsymbol{r}, t)=\sum_{t}\left[\boldsymbol{E}_{\boldsymbol{k}_{f} \omega_{j}} \exp \left\{1\left(\omega_{j} t-\boldsymbol{k}_{j} \cdot \boldsymbol{r}\right)\right\}+\mathrm{cc}\right] / 2
$$

Here $\mathrm{c} c$ stands for the complex conjugate and the summation runs over all values $\left(\boldsymbol{k}_{j}, \omega_{j}\right)$ of the plane waves Then considering for the moment only linear polarization, it follows from eqns (1) and (2) that

$$
\begin{aligned}
\boldsymbol{P}(\boldsymbol{k}, \omega) \equiv & \epsilon_{0} \int \mathrm{d} \boldsymbol{r} \mathrm{d} t \boldsymbol{P}(\boldsymbol{r}, t) \exp \{1(\boldsymbol{k} \quad \boldsymbol{r}-\omega t)\} \\
= & \epsilon_{0} \int \mathrm{d} t \mathrm{~d} \boldsymbol{r} \mathrm{d} t_{1} \mathrm{~d} \boldsymbol{r}_{1} \chi^{(1)}\left(\boldsymbol{r}-\boldsymbol{r}_{1}, t-t_{1}\right) \boldsymbol{E}\left(\boldsymbol{r}_{1}, t_{1}\right) \\
& \times \exp \{1(\boldsymbol{k} \cdot \boldsymbol{r}-\omega t)\} \\
= & \epsilon_{0} \chi^{(1)}(\boldsymbol{k}, \omega) \boldsymbol{E}(\boldsymbol{k}, \omega)
\end{aligned}
$$

In eqn (3) the $3 \times 3$ matrix $\chi^{(1)}(\boldsymbol{k}, \omega)=\int \chi^{(1)}(\boldsymbol{r}, t)$ $\times \exp (\iota \boldsymbol{k} \cdot \boldsymbol{r}-\imath \omega t) \mathrm{d} \boldsymbol{r} \mathrm{d} t$, and we have utllized the fact that the Fourier transform of a convolution may be expressed in terms of the product of the transforms

The higher-order terms contributing to the polarization can be obtained in a similar way, $1 \mathrm{e}$, by substitution of eqn (2b) into eqn (1) and collecting terms which contribute to the same $\boldsymbol{P}(\boldsymbol{k}, \omega)$ term For example, if we consider third-order NLO effects with one incoming plane wave in an isotropic medium, and the electric field, $E_{1}\left(\boldsymbol{k}_{1}, \omega_{1}\right)$, polarized along the 1-axis, it follows that there are two different polarization terms

$$
\begin{aligned}
& P_{1}^{(3)}(\boldsymbol{r}, t)= \epsilon_{0} \chi_{1111}^{(3)}\left(\boldsymbol{k}=\boldsymbol{k}_{1}+\boldsymbol{k}_{1}+\boldsymbol{k}_{1},\right. \\
&\left.\omega=\omega_{1}+\omega_{1}+\omega_{1}\right) \\
& \times\left[E_{1 k_{1} \omega_{1}}^{3} \exp \left\{31\left(\omega_{1} t-\boldsymbol{k}_{1} \cdot \boldsymbol{r}\right)\right\}+\mathrm{cc}\right] / 2 \\
& P_{1}^{(3)}(\boldsymbol{r}, t)=3 \epsilon_{0} \chi_{1111}^{(3)}\left(\boldsymbol{k}=\boldsymbol{k}_{1}+\boldsymbol{k}_{1}-\boldsymbol{k}_{1}\right. \\
&\left.\omega=\omega_{1}+\omega_{1}-\omega_{1}\right) \\
& \times\left|E_{1 k_{1}{ }_{111}}\right|^{2} E_{1}(\boldsymbol{r}, t)
\end{aligned}
$$

It is of importance to note that the two third-order susceptibilities in eqn (4) arise from different Fourier transforms of the same susceptibility in $r, t$-space The first term of eqn (4) describes the frequency tripling, which is of importance for third-harmonic generation (THG), and the second term leads to a polarization, phase-matched to the incoming electric field, giving rise to an intensitydependent refractive index (IDRI, see below) The degeneracy factors 1 and 3, occurring in eqn (4), are the number of ways in which these products of electric fields can be made, and ensure that the parameters $\chi^{(3)}$ are continuous as a function of the arguments $\boldsymbol{k}$ and $\omega$ [2] For example, this way 
TABLE 1 Susceptibilities for various NLO effects and its applications The abbrevations EO, SHG and THG stand for electro-optic, secondand thurd-harmontc generation, respectively

\begin{tabular}{lll}
\hline Susceptibility & Effect & Application \\
\hline$\chi^{(2)}(\omega=\omega+0)$ & electro-optic (EO) effect & EO modulation of light \\
$\chi^{(2)}(2 \omega=\omega+\omega)$ & frequency doubling & SHG \\
$\chi^{(2)}\left(\omega_{1}=\omega_{2}-\omega_{3}\right)$ & frequency mixing & down conversion \\
$\chi^{(3)}(0=0+\omega-\omega)$ & quadratic EO effect & EO modulation of light \\
$\chi^{(3)}\left(\omega_{1}=\omega_{1}+\omega_{2}-\omega_{2}\right)$ & Raman effect & all-optical devices \\
$\chi^{(3)}(\omega=\omega+\omega-\omega)$ & a c Kerr effect & all-optical devices \\
$\chi^{(3)}(2 \omega=0+\omega+\omega)$ & electric-field-induced SHG & SHG \\
$\chi^{(3)}(3 \omega=\omega+\omega+\omega)$ & frequency tripling & THG \\
\hline
\end{tabular}

$$
\begin{aligned}
& \lim _{\boldsymbol{k}_{1} \omega_{1} \rightarrow k_{\omega}} \chi^{(3)}\left(\boldsymbol{k}=\boldsymbol{k}+\boldsymbol{k}_{1}-\boldsymbol{k}_{1}, \omega=\omega+\omega_{1}-\omega_{1}\right) \\
& =\chi^{(3)}(\boldsymbol{k}=\boldsymbol{k}+\boldsymbol{k}-\boldsymbol{k}, \omega=\omega+\omega-\omega)
\end{aligned}
$$

A lot of different non-linear phenomena are known today, some of these are given in Table 1

The polarization affects the propagation of the light, which may be seen using Maxwell's equations Depending on the nature of the effect, different approaches may be used For non-automatically phase-matching effects, such as the second-harmonic generation (SHG), the so-called SVEA (slowly varying envelope approximation) is often used [2] We shall treat here in some more detall the first- and third-order (IDRI) effects In order to keep things simple, we assume an isotropic current- and charge-free non-magnetic matcrial Then Maxwell's cquations lead to the following form of the wave equation

$$
-\nabla^{2} \boldsymbol{E}(\boldsymbol{r}, t)+\frac{1}{c^{2}} \frac{\partial^{2} \boldsymbol{E}(\boldsymbol{r}, t)}{\partial t^{2}}=-\mu_{0} \frac{\partial^{2} \boldsymbol{P}(r, t)}{\partial t^{2}}
$$

For a single plane wave with an electrical field along the 1-axis, it may be deduced from eqns (1)-(4) that the solutions of eqn (6) are of the form

$E_{1}(\boldsymbol{r}, t)=\left[E_{1 k_{t}} \exp \left\{\mathbf{1}\left(\omega t-n \boldsymbol{k}_{0} \cdot \boldsymbol{r}\right)\right\}+\mathrm{cc}\right] / 2$

with $\left|k_{0}\right|=\omega / c$, and $n^{2}=1+\chi^{(1)}+3 \chi_{111}^{(3)}\left|E_{1 k \omega}\right|^{2}$

Equation (7) is the expression for the ideal Kerr law non-linearity, neglectıng higher-order effects leading to, e $g$, saturation of the NLO effect $A$ notation often encountered in the literature is

$n=n_{0}+n_{2, E}|E|^{2}=n_{0}+n_{2} S$

with $n_{2 E} \simeq 3 \chi_{111}^{(3)} /\left(2 n_{0}\right)$ Here $S$ is the energy flow (in $\mathrm{W} / \mathrm{m}^{2}$ ) of the beam and from standard theory it follows that

$$
n_{2 s}=\frac{2}{n_{0} \epsilon_{0} c} n_{2 E}
$$

Inspecting eqns (1-3), it follows that, if the susceptibilities $\chi^{(n)}\left(r-r_{1}, \quad\right)$ are vanishing unless $\left|r-r_{\imath}\right| \ll \lambda$, with $\imath=1, \quad, n$, and $\lambda$ is the wavelength of the light, the Fourier transforms are approximately $k$-independent This situation may be met if the NLO material consists of relatively small non-interacting molecules, but, for example not in semiconductors if mobile charge carriers or excitons are involved Such non-local effects generally hamper the solution of the Maxwell equations $\mathrm{in}$, e $\mathrm{g}$, the presence of optical waveguides and may also affect device performance [3] If the above approximation holds, which is often assumed in simulations, the argument $k$ in eqn (7) may be omitted, thus facilitating the solution of the wave equation for a propagating electric-field pattern, generally consisting of a summation over plane waves The latter holds for non-uniform media, e $\mathrm{g}$, media in the presence of waveguides

\section{Third-order NLO materials}

\section{Theory}

Third-order NLO effects give rise to a lot of interesting phenomena, as depicted in Table 1 For applications in all-optical processing at a single frequency, the optical Kerr effect (or IDRI) is of special importance This Section concentrates mainly on this effect, although part of the discussion holds equally well for related effects such as Raman-active non-linearities or the third-order electro-optic effect

To start the discussion on the IDRI, we believe that it is instructive to consider a three-level 


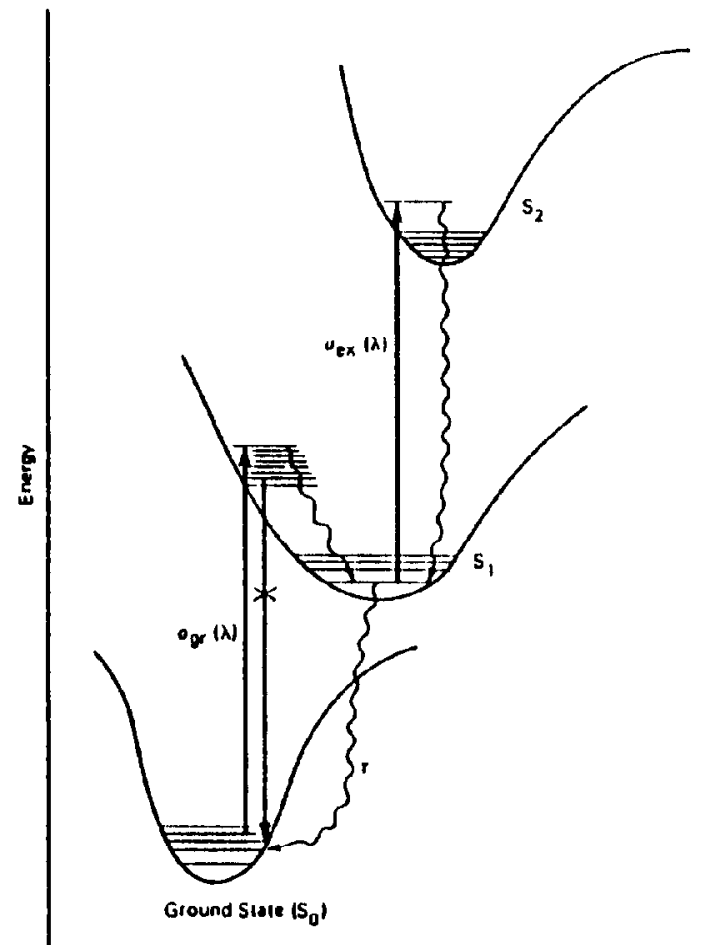

Nuclear Configuratuon Coordunave

Fig 1 Schematic of the electronic energy levels of a saturable absorber

molecule Figure 1 shows a diagram of a molecule having three electronic levels [4] If the molecule gets excited by incident light, the state $S_{1}$ gets populated at the expense of the ground state It is of importance, in order to understand the underlying mechanism of the IDRI, to note that, by the population of $S_{1}$, the absorption of the molecule will change only if the transitions $S_{1} \longrightarrow S_{2}$ differ from that of the ground state, $1 \mathrm{e}$, if state $S_{1}$ has a different polarizability compared with the ground state Due to changes in the absorption coefficient, the real part of the refractive index also changes The relation between the real and imaginary parts of the refractive index is described by the Kramers-Kronig relations Above we have in fact discussed the resonant case Similar conclusions hold for the non-resonant case, where the excitations do not occur but where the excited states are mixed into the ground state by the optical field

One of the earliest attempts to find analytical expressions for the third-order susceptibility of polymers uses a model in which $2 M$ electrons are confined to a one-dimensional box of length $2 L$, oriented along the $z$-axis [5] Using perturbation theory, the result for large $L$ is

$\gamma_{z z z z}=\frac{256 L^{10}}{45 a_{0}{ }^{3} e^{2} \pi^{6} M^{5}}$

Here $a_{0}$ is the Bohr radius and $\gamma$ is the second hyperpolarizability, for which $\gamma \cong \chi^{(3)} / N$ approx1mately holds, $N$ being the particle density The result has been obtained by taking the fourth derivative of the ground-state energy with respect to the electric $d c$ field So the result should correspond to the non-resonant value and predicts the right order of magnitude for polyenes and cyanmes [5] Assuming $M \propto L$, it follows that $\gamma \propto L^{5}$ In eqn (10) the strong dependence of $\gamma$ on the chain length $L$ can be attributed both to an increasing dipole strength and a decreasing energy gap as a function of $L$

For long polymeric chains, the box-model described above predicts non-transparency, which can indeed be observed for some classes of polymers, such as cyanınes Polyenes, however, remain transparent even for very long chain lengths due to a Peierls transition [6] In the theoretical model used in ref 5 this has been accounted for by using a sinusoidal potential, leading to a non-vanishing band gap, $E_{\mathrm{g}}$ Using an extension of this model, Sauteret et al [7] found the following relation for infinite polymeric chains of polyenes

$\chi_{z z z z}^{(3)}=\frac{2^{5}}{45} \pi^{2} \cdot \frac{e^{10}}{\sigma}\left(\frac{a_{0}}{d}\right)^{3}\left(\frac{1}{E_{\mathrm{g}}}\right)^{6}$

Here $\sigma$ is the cross-sectional area per chain and $d$ is the average $\mathrm{C}-\mathrm{C}$ distance

Analytical expressions of the third-order susceptibility as a function of photon energy, $\omega$, have been published $[6,8-10]$ In refs 6 and 8 the authors use the Keldysh non-equilibrium Green's function method, with the band gap as the only adjustable parameter The other methods are based on the Genkın-Wednis method [9] and a new method that includes damping effects [10] The results are in excellent agreement with measurements of the THG as a function of $\omega$ in trans-polyacetylene, and predict the correct order of magnitude for the IDRI Detalled measurements of the latter for comparison with the calculations are not avalable at the moment From the analytical expressions [6], we deduce a dependence $\chi^{(3)} \propto 1 / E_{\mathrm{g}}{ }^{6}$ for the $\mathrm{d} \mathrm{c}$ limit, in agreement with the result given in eqn (11) 
Various different $a b$ initio methods are used today to calculate non-linear susceptibilities We shall discuss briefly two of the most frequently used

(1) The hyperpolarizability is obtained from the differential of the ground-state energy with respect to a static electric field [11] Here two approaches can be followed In the first, the self-consistent field (SCF) equation is solved in the presence of a finite field, and $\alpha$ and $\gamma$ are obtained by finite differencing In the second, the differentiation is performed analytically, this involves solutions of coupled perturbed Hartree-Fock solutions [12]

(11) The hyperpolarizability is calculated using the so-called direct summation method [13] Here the matrix elements for dipole transitions and the energies of excited states are computed by some SCF method, by substituting these quantities into analytical expressions for the susceptibilities (the electronic contributions to) the latter are obtained

Results obtained with method (1) [11] on transpolacetylenes, $\mathrm{C}_{2 p} \mathrm{H}_{2 p+2}, p=1$, ,5, show a power-law dependence $\gamma \propto L^{5}$ for $p \geqslant 2$, in remarkably good agreement with eqn (10), obtained in a relatively simple way A similar powerlaw dependence has been found [13] for $\gamma(3 \omega=\omega+\omega+\omega)$ of trans- and cts-polyacetylene at $\omega=065 \mathrm{eV} \gamma \propto L^{47}$

Experiments [14], measuring the THG of sidechain-substituted polymers as a function of the number of double bonds, $n_{\mathrm{db}}$, show an exponential behaviour $\chi^{(3)} \propto n_{\mathrm{db}}{ }^{32}$ This is in rather good agreement with the results mentioned above and also with results using the two-level model [14]

The theories outlined above do not take into account the effects due to electron correlation These effects hamper the calculations of $\chi^{(3)}$ but are shown, both experimentally [15] and theoret1cally [16], to be of great importance in organics, and also in a lot of other materials, as may be expected

\section{Materials}

Today, a lot of materials showing more or less strong NLO third-order effects are known The characteristics of some of these materials are depicted in Table 2 Below we shall discuss the most promising materials with respect to applications in fast all-optical devices MQWS (multiple quantum well structures) [17], embedded noble metal and semiconductor $\left(\mathrm{CdS}_{x} \mathrm{Se}_{1-x}\right)$ particles of nanometre size and polymers

QWS consist of ultrathin (typically $100 \AA$ ) layers of semiconductor materials having physical compatibility but a different composition Thus the band gap may be modulated in a direction perpendicular to the layers, leading, for a sufficiently large modulation, to a confinement of the carriers Thus the band structure is $2 \mathrm{D}$ (two $\mathrm{d}_{\mathbf{l}}$ mensional) in nature

As in 3D semiconductors, Coulomb attraction leads to bound electron-hole $(\mathrm{e}-\mathrm{h})$ states or exc1tons The effect of the confinement leads to an artificial decrease of the $\mathrm{e}-\mathrm{h}$ distance, and thus to an increase of the binding energy of the excitons, and also to an enhancement of the exciton density of states The latter leads again to an increase of the oscillator strength for excitations to exciton states

This large binding energy leads to more stable exciton states, which has a drastic effect on the complex refractive index due to both phase-space filling and screenıng effects The third-order NLO effects in MQWS of GaAs/AlGaAs are extremely

TABLE 2 Experimental values of $n_{2}$ at room temperature, the corresponding reaction time and the saturation value of the refractive index change (if known), $\Delta n_{\text {sat }}$ The wavelength, $\lambda$, is given only if there is a unique wavelength (region) for the IDRI The abbreviations used are $\mathrm{o}=$ orientational, $\mathrm{t}=$ thermal, $\mathrm{e}=$ electronic and $\mathrm{ph}=$ phase-space filling

\begin{tabular}{|c|c|c|c|c|c|c|}
\hline Material & $n_{2}(\mathrm{~m} / \mathrm{V})^{2}$ & $\tau(s)$ & $\Delta n_{\text {sat }}$ & $\lambda(\mu \mathrm{m})$ & Effect & Ref \\
\hline MBBA (hq cr) & $10^{-12}$ & $10^{-6}$ & 01 & & $o / t$ & 18 \\
\hline $\mathrm{ZnS}$ & $10^{-13}$ & $10^{-6}$ & & & $t$ & 18 \\
\hline Doped glass & $-4 \times 10^{-18}$ & $10^{-10}$ & 0001 & & e & 19,20 \\
\hline Polydiacetylene & $10^{-18}$ & $10^{-12}$ & & & $\mathrm{e} / \mathrm{ph}$ & 21 \\
\hline Polythiophene & $10^{-17}$ & $<10^{-12}$ & & & e & 22 \\
\hline
\end{tabular}


large (see Table 2) In this material the lifetıme of excitons is about $1 \mathrm{ps,}$, experiments [17] have shown that after a strong excitation pulse, having a photon energy equal to that of the exciton transition, the absorption coefficient (at that photon energy) drops within 1 ps and recovers slowly The relatively long recovery time ( $1 \mathrm{~ns}$ ) is due to interactions with free electrons and holes, and is quite disadvantageous for applications

Recent experiments on small structures in MQWS indicate that the slow recovery times can be overcome by diffusion of the electrons and holes to the surface followed by recombination [24]

Quite interesting, and very promising for appl1cations in all-optical logic and memory devices, is the behaviour of MQWS of GaAs/AlGaAs in the presence of an electric field perpendicular to the layers [17] Then, the exciton wavefunctions change quite drastically, leading to a change of the optical properties, such as a shift of the exciton peak This effect is often referred to as the quantum-confined Stark effect, and is used in so-called SEEDs (self electro-optic effect devices) The latter are used today in laboratory experiments on opt1cal computers as logic and memory devices, operating at low switching energies [25]

In small $(1-10 \mathrm{~nm})$ noble-metal particle suspensions the NLO effects are attributed to the quantum size effect (QSE), $1 \mathrm{e}$, to the confinement of the electrons, although thermal effects cannot be ruled out [26] Usually, thermal effects lead to large reaction times, but for such small particles the heat diffusion is a fast $(\approx 1 \mathrm{ps})$ effect

As mentioned above, the QSE leads to a large increase of the third-order NLO effects for small metal partıcles An extra enhancement can be obtained if small noble metal particles are used, at frequencies of light close to the plasmon frequency This may be explained as follows For small (s1ze « wavelength) particles the electrostatic approximation may be used The relation between the electric field of the radiation in a particle, $E_{\mathrm{p}}$, and that of the incoming field, $E_{\mathrm{m}}$, is then given by [27]

$$
E_{\mathrm{p}}=\frac{3 \epsilon_{\mathrm{m}}}{\epsilon_{\mathrm{p}}+2 \epsilon_{\mathrm{m}}} E_{\mathrm{m}}
$$

Here $\epsilon_{\mathrm{m}}$ and $\epsilon_{\mathrm{p}}$ are the dielectric constants of the host medium and the metal particles, respectıvely For noble metals the real part of $\epsilon_{\mathrm{p}}$ is negative for frequencies below the plasmon frequency, so that, for a suitably chosen host and frequency, we may find

$\operatorname{Re}\left(\epsilon_{\mathrm{p}}+2 \epsilon_{\mathrm{m}}\right)=0$

This is the condition for plasmon resonance If eqn (13) holds, this will lead to a strong enhancement (orders of magnitude) of the electric field inside, and in the vicinity of, the noble metal particles, and therefore to a strong increase of the third-order NLO effects $[26,27]$ It has been shown [27] that using the expression for the IDR I (see Section 2), eqn (12) may have more than one solution for $E_{\mathrm{p}}$ at a given $E_{\mathrm{m}}$, leading to an intrinsic optical bistability, thus allowing for memory devices on a sub-micron scale

The above discussion holds as well for noble metal particles embedded in a NLO host, but here the conditions may be chosen such that a much stronger enhancement of the NLO effects is obtained For pure thermo-optic materials, such as $\mathrm{ZnS}(\mathrm{e})$, the third-order NLO effects are large but slow $(\cong \mu \mathrm{s})$ on a picosecond tıme scale We remark that, using such a material as a host for small noble metal particles, advantage may be taken of both the plasmon enhancement effect in the vicinity of the metal particles and the fact that heat will diffuse away from such a small particle within a few picoseconds In this way strong NLO fast-reacting materials can be obtained, suitable for applications in the field of optical phase conjugation, opto-optic devices and small-sızed memory elements, working at relatively low intensities of the light

Other suggestions in this field have been presented [28] and consist of nanoparticles having a NLO core of, e g, polymers Calculations [28] show that the presence of such a core leads to an increase of the non-linearity and to a lower onset of optical bistability

With respect to second-order NLO effects, organics can compete with anorganic crystals Inspecting Table 2, it follows that a similar remark cannot be made with respect to the IDRI, the value of $n_{2}$ in polymers is not better than $10^{-17} \mathrm{~m}^{2} / \mathrm{V}^{2}$ For practical applications in alloptical devices this value is far too low and MQWS seem to be favourable here, at least at the moment

The advantages of polymers are that these materials have fast response times and can be tailored to have (1) good transmission properties in the 
desired optical frequency range and (11) higher values of $n_{2}$ With respect to the latter, there is a need for systematic experimental and theoretical investigations on which an organic group or combination of groups may lead to an enhancement of the effect, and also to the influence of the chain length

\section{Characterization}

In order to utılize NLO effects in optıcal devices and also for material characterization, it is advantageous to use optical waveguides as (1) this leads to a strong concentration of the optical field, and (11) the propagation of modes is diffractionless, leading to long interaction lengths Accurate measurements of NLO effects are of importance both for device modelling and in order to get insight into the material properties Many different characterization methods, mostly based in interferometric effects, may be used to measure the reaction tıme and magnitude of third-order NLO effects [29] In this Section we shall discuss some methods based on prism coupling into planar structures for the determination of the magnitude of the IDRI and also of the second- and third-order electro-optic effect

A very sensitive way to detect small index changes consists of coherent detection of the variation in the coupling efficiency of a signal beam, induced by a modulated pump beam Choosing a coordinate system as given in Fig 2, the differential equation for the coupling of both the signal and the pump beam is given by [30]

$\frac{\mathrm{d} A_{\mathrm{m}}}{\mathrm{d} z}=-\left(K_{\mathrm{m}} k_{0}+\beta_{\mathrm{m} 1}\right) A_{\mathrm{m}}+t_{\mathrm{m}} K_{\mathrm{m}} k_{0} E_{0} \exp \left(1 \Delta \beta_{\mathrm{r}} z\right)$

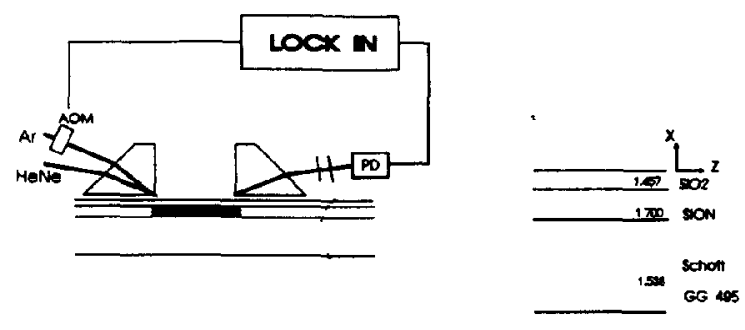

Fig 2 Experımental set-up for the coherent detection of modulation of the coupling efficiency of the signal beam ( $\mathrm{HeNe}$ ) by a pump beam $\left(\mathrm{Ar}^{+}\right) \mathrm{AOM}=$ acousto-optic modulator and $\mathrm{PD}=$ photodetector
Here $E_{0}$ is the amplitude of the electrical component of the incoming beam at the prism base, $A_{\mathrm{m}}$ is the amplitude of the model field which is related to the electric modal field, $E_{\mathrm{m}}$, by

$E_{\mathrm{m}}(x, z)=A_{\mathrm{m}}(z) \xi_{\mathrm{m}}(x) \exp \left\{-1 \beta_{\mathrm{m} \mathrm{r}} z\right\}$

$\Delta \beta_{\mathrm{r}}=k_{0} n_{\mathrm{p}} \sin (\theta)-\beta_{\mathrm{m} \mathrm{r}}$, where $k_{0}$ is the wavevector, $n_{\mathrm{p}}$ is the refractive index of the prism and $\theta$ is the angle of incidence on the prism base $\beta_{\mathrm{m}}=\beta_{\mathrm{m} \mathrm{r}}+1 \beta_{\mathrm{m} \text { I }}$ is the propagation constant of the mode In eqn (15) the field shape $\xi$ is defined such that $\left|A_{\mathrm{m}}\right|^{2}$ represents the power of the mode per unit length in the $y$-direction The coupling constants $K_{\mathrm{m}}$ and $t_{\mathrm{m}}$ are interrelated [31] and may be obtained, for a given structure, in a way described by Tien and Ulrich [32] (see also [33])

In the presence of a third-order non-linearity, the propagation of the mode becomes power dependent and, neglecting any power-dependent changes of the modal field, it follows [35] that

$\beta_{\mathrm{m}}(z)=\beta_{\mathrm{m}}^{0}+\beta_{2}|A(z)|^{2}$

The relation between $\beta_{2}$ and the material parameters can be determined using standard perturbation theory [34]

With the above it follows that, for prism coupling with a single beam, the coupling efficiency is power dependent The NLO properties of the involved materials can be measured in this way [36]

In the presence of a modulation of the signal beam by a stronger beam, either at a different wavelength or exciting a different mode, coherent detection techniques can be used $[21,37]$, see Fig 2 Such a set-up leads to a very sensitive detection of intensity-induced changes in the refractive index, $\Delta n \geqslant 10^{-8}$, allowing also for measurements of saturation effects, Raman processes $\left\{\chi^{(3)}\left(\omega=\omega+\omega_{1}-\omega_{1}\right)\right\}$, non-1deal Kerr-law behaviour and the performance of time-resolved pump probe processes Angle-dependent measurements of the out-coupled power at the output prism of the signal beam and of its modulation by the pump beam are shown in Fig 3 The coupling angle of the pump beam remains unchanged Changes in both the real and imaginary parts of the refractive index can be revealed $[21,37]$

A similar modulation technıque may also be used to determine both the second- and thirdorder EO coefficients, an ATR configuration was used for the experiments in refs 38 and 39 

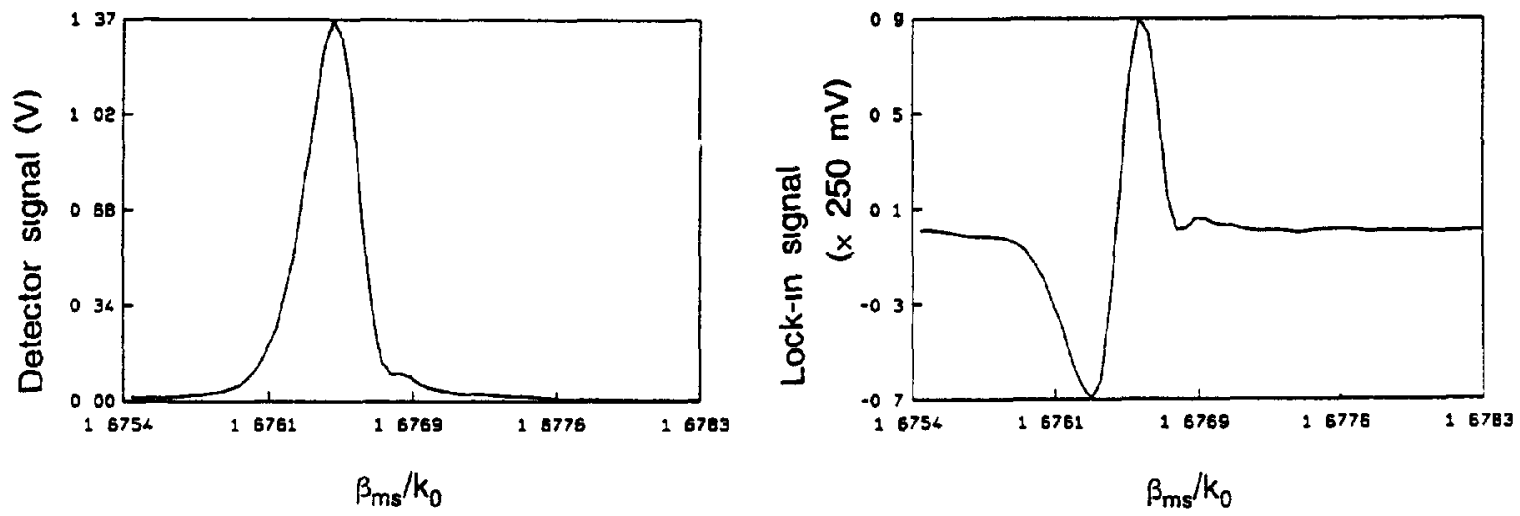

Fig 3 Experimental results obtained with the equipment depicted in $F_{1 g} 2[37] \beta_{\mathrm{m} \mathrm{s}} / k_{0}$ is the effective index of the mode excited by the signal $(\mathrm{HeNe})$ beam

\section{All-optical devices}

A large variety of integrated optics devices utilızing the IDRI have been proposed and partly realized and tested in the last fifteen ycars The cffects on which the performance of these devices rest may roughly and non-exclusively be subdivided into

(1) a change in the modal propagation constant $(\Delta \beta)$, leading to a phase shift $(\Delta \varphi)$, made effective by interference with some other beam or mode, occurring for example in Mach-Zehnder interferometers (MZI) and distributed (prism or gratıng) couplers,

(11) a $\Delta \beta$ (and $\Delta \varphi$ ) for a device with a performance depending critically on some dimension of the device, such as in a Fabry-Perot (FP) etalon or a gratıng mode-converter,
(111) a change in the (modal) refractive index, having the order of magnitude of the linear (modal) index differences in the device, such as occurring in the case of spatial soliton emission, NLO Y- and X-junctions and optical limiters,

(1v) devices utilizing, besides the effect mentioned in (11), the intensity-dependent cross-phase modulation between different modes, e $\mathrm{g}$, logic switches,

(v) optically bistable (OB) clements such as FP etalons, gratıngs and distributed couplers, where the bistability is caused by some feedback mechanism,

(vi) hybrid devices working not solely in the optical domain, e g, SEEDs

In Table 3 we have given a survey of a number of more or less well-known third-order non-linear

TABLE 3 An overview of third-order non-linear devices, indicating whether a given performance has been reported or may be expected ( $y$ ) or not $(n)$, and the corresponding references Part of the devices ( $\mathrm{g}, \mathrm{X}, \mathrm{Y}$-junction) act as a memory only in the presence of diffusion of the NLO effect, such as inducing the necessary feedback

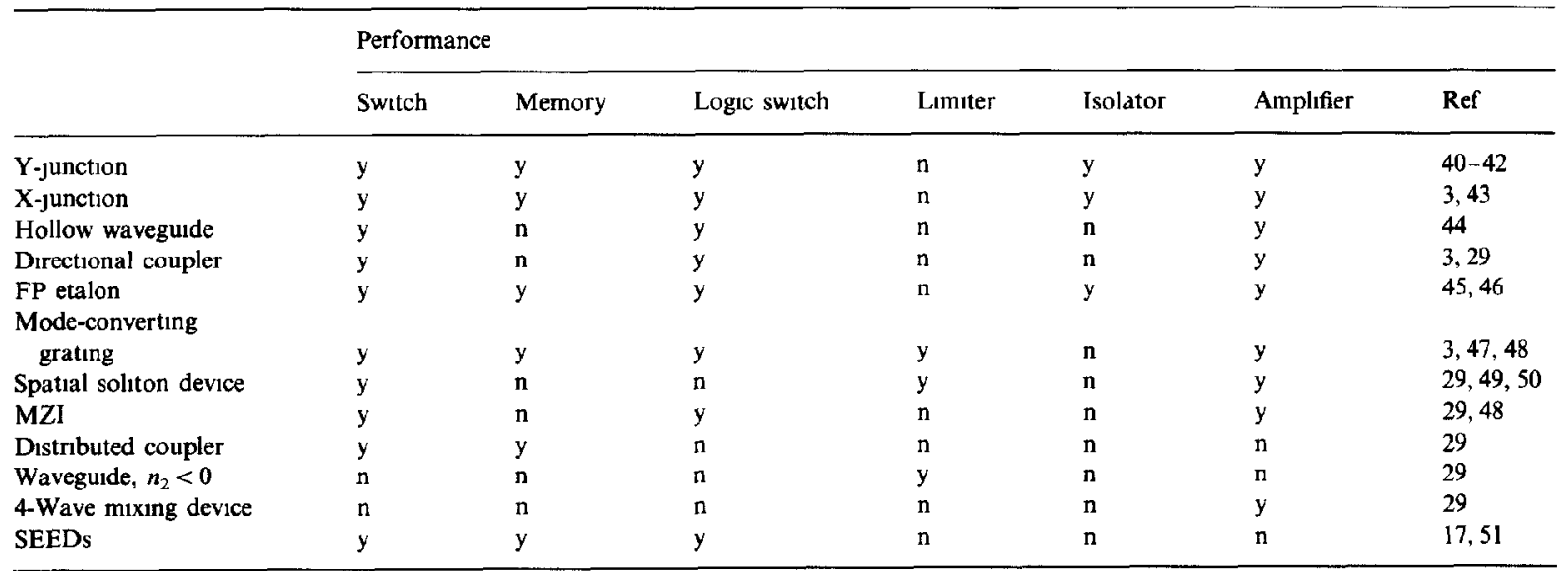


devices, for completeness SEEDs are also mentioned, although their performance is based on higher-order NLO effects SEEDs and other hybrid devices are of interest due to the low optical power required for switching Recently the operation of arrays of symmetric SEEDs [51], an optical parallel processor using spatial light modulators [52] and an optical tristable device using an integrated phototransistor and laser diode [53] have been demonstrated Although such hybrid devices have great merits, certainly if the parallelism is exploited, these devices are electronically assisted and so limited in their switching speed

For ultrafast serial processing of information, all-optical devices with their potentially short switching times ( $\leqslant 1 \mathrm{ps)}$ ) are needed In telecommunications such devices can be used for routing by decoding the address headers and also to provide rapid access to a network Demonstrations of and discussions on such devices can be found in the recent literature $[54,55]$

For detailed discussions on the devices given in Table 3 the interested reader is referred to the papers indicated Here we shall treat in some more detal the merits of devices consisting of a single (non)-linear asymmetrical Y-junction and a device consisting of a combination of that, the non-linear Mach-Zehnder interferometer (NMZI)

Y-junctions are of interest in integrated optics due to the versatility of the functions that can be performed with these structures and the relaxed fabrication tolerances (as compared to directional couplers, for example) Burns and Milton [40] have shown that $\mathrm{Y}$-junctions can act etther as power splitters or mode splitters Then, from reciprocity, it can be understood that $\mathrm{Y}$-junctions can also be used as mode converters and signal combiners, provided that the conversion to radiation modes in the Y-junction structure is negligible The requirement for this is that the branching angle is sufficiently small, so that the modal field propagation is adiabatic In order to perform BPM (beam propagation method [56-59]) simulations, it is convenient to approximate the 3D structure by a $2 \mathrm{D}$ one on using the effective-1ndex method (EIM [34])

In Fig 4 a 2D picture is given of an asymmetric Y-junction It was shown [40] that asymmetric Y-junctions consisting of one bimodal input and two non-1dentical monomode output channels can act as mode splitters As a key parameter, a mode conversion factor (MCF) can be defined by

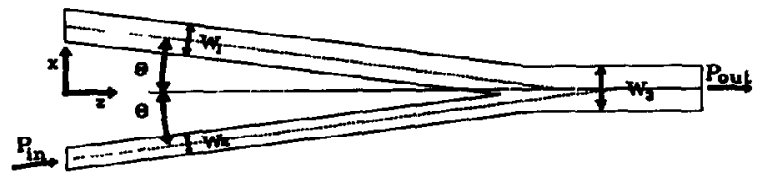

Fig 4 Schematic of the considered Y-junction

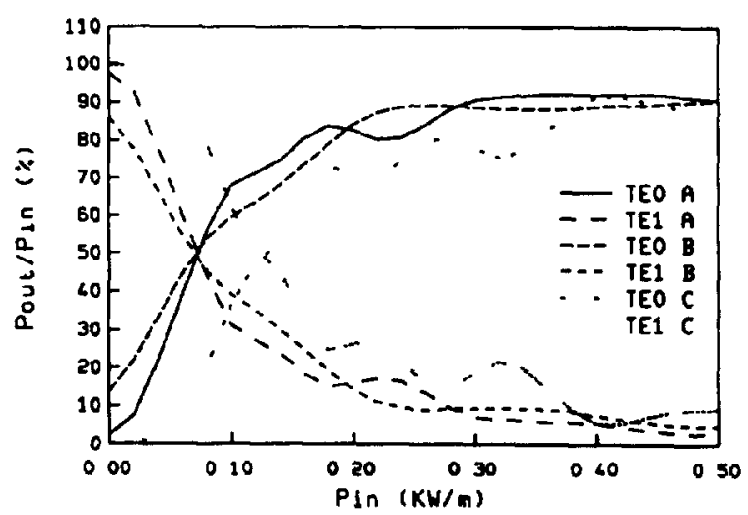

Fig 5 Switching behaviour of the Y-junction depicted in Fig 4 The parameters used for the simulations are background index, $n_{\mathrm{b}}=159$, waveguide index, $n_{\mathrm{s}}=16$, homogeneous non-linearity, $n_{2 E}=10^{-13} \mathrm{~m}^{2} / \mathrm{V}^{2}$, and $\lambda=1 \mu \mathrm{m}$ For the widths we have used $w_{1}=225 \mu \mathrm{m}$ (cases $A$ and $B$ ) and $25 \mu \mathrm{m}$ (case C) and $w_{2}=2 \mu \mathrm{m}$ The crossing angle was $2 \theta=02035^{\circ}$ ( $A$ and $C$ ) and $04035^{\circ}$ (case B)

$\mathrm{MCF}=\frac{N_{2}-N_{1}}{\operatorname{tg}(2 \theta)\left(\bar{N}^{2}-n_{3}^{2}\right)^{1 / 2}}$

where $\theta$ is the half branching angle, $n_{3}$ is the index of refraction of the medium between the two branching angles, $\bar{N}=05\left(N_{1}+N_{2}\right)$ and $N_{1}$ and $\mathrm{N}_{2}$ are the effective indices of waveguides 1 and 2 at infinite separation For $|\mathrm{MCF}| \gg 044$ it was found that the Y-junctions act as mode splitters, enabling efficient power transfer from the input channel mainly to one of the output channels, whereas $|\mathrm{MCF}| \ll 044$ leads merely to a division of power over both output channels

From reciprocity, it can be understood that a $\mathrm{Y}$-junction with large MCF and low conversion to radiation modes can also be operated in the opposite direction, $1 \mathrm{e}$, light launched in one of the two inputs is efficiently coupled to the fundamental or first-order mode of the output channel

It is clear from eqn (17) that the MCF, and thus the behaviour of the Y-junction, is power dependent in the NLO case This can also be seen from the results of the BPM simulations depicted in $\mathrm{F}_{1 \mathrm{~g}} 5$ Here we have used an overall non-linear1ty $n_{2}=10^{-13} \mathrm{~m}^{2} / \mathrm{V}^{2}$, and effective indices $n_{\mathrm{b}}=159$ 
and $n_{\mathrm{g}}=16$ for the background and the waveguiding sections, respectively The wavelength is $1 \mu \mathrm{m}$, and the fundamental mode is launched into the lower branch at the left of Fig 4 From Fig 5 it follows that at low power levels, the field is converted into a first-order mode, whereas at higher power levels the output is mainly the fundamental mode By placing another (mode-splittıng) Y-junction, but now a linear one, somewhere further on in the structure, the device acts as an all-optical switch

The device is non-reciprocal, as a fundamental mode launched at the right of the junction (see Fig 4) will leave it mainly (more than $999 \%$ for not too high power levels [60]) through the upper part Thus we suggest that it can be used as an optical 1solator of a pulsed laser, at least if things can be arranged such that a reflected pulse cannot coincide with a new incoming pulse in the effective part of the junction

We also expect that the device may be used as an all-optical amplifier and a logic gate Similar interestıng features have also been described [43] for an X-junction

The switching curve becomes much steeper, compared to that of Fig 5, if a Y-junction is used for which one of the two branches is a hollow waveguide A hollow waveguide is a waveguide that becomes gurding only in the presence of a certain threshold intensity, due to a positive nonlinearity In Fig 6 results are shown for a Y-junc-

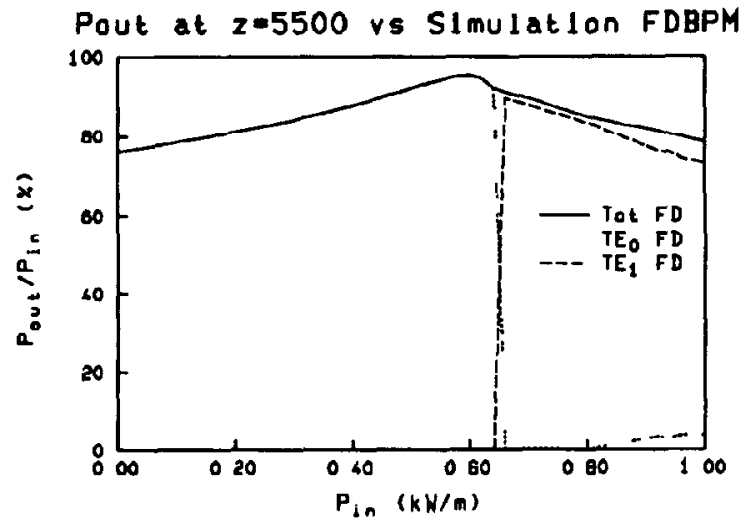

Fig 6 Switching behaviour of a Y-junction having one branch consisting of a hollow waveguide, also being the only NLO part of the structure Here a fundamental mode is launched into the part consisting of a single branch, and the power in the $\mathrm{TE}_{1}$ mode represents the power leaving the junction through the hollow branch The parameters are similar to the ones given in the caption of Fig 5 except $n_{\mathrm{g}}=1595$ and $2 \theta=01$

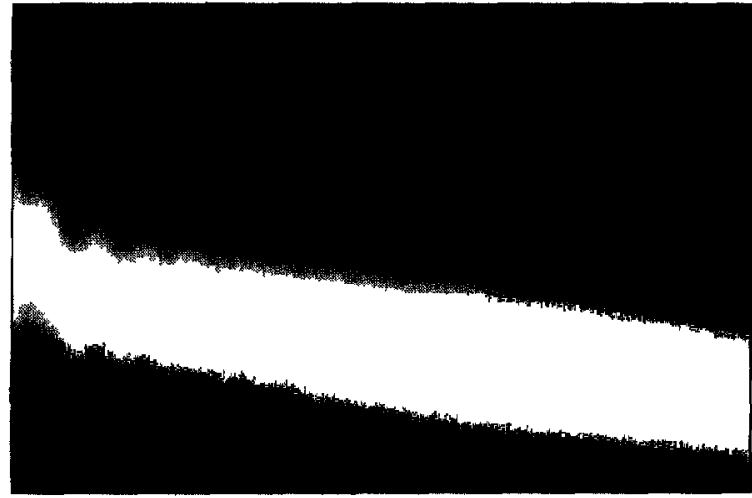

(a)

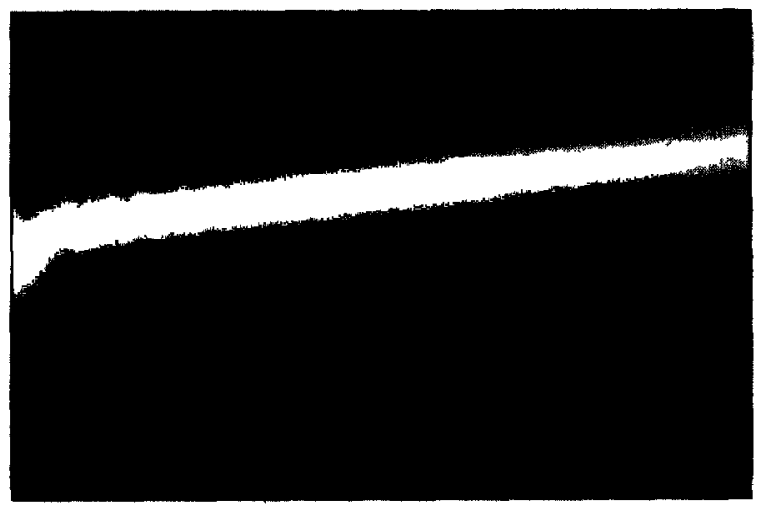

(b)

Fig 7 BPM simulations of ficld propagation in the $Y$-junction described in the caption of Fig 6 at power levels just below ((d) $\left.P_{\mathrm{in}}=640 \mathrm{~W} / \mathrm{m}\right)$ and above ((b) $\left.P_{\mathrm{in}}=660 \mathrm{~W} / \mathrm{m}\right)$ the critical power The fundamental mode is launched at the left of the picture

tion where the hollow branch is the only non-linear part of the structure and a fundamental mode is launched into that part of the structure consisting of a single branch The hollow branch has, in the active region of the junction, a linear refractive index equal to that of the background Above a certain threshold (spatial) soliton emission occurs into the hollow (upper) branch (see Fig 7) The latter corresponds to the $\mathrm{TE}_{1}$ mode, see Fig 6

Interferometric measurements have for a long tıme played an important role in the history of free-space optical measurements Small changes in optical path length can be measured with accuracies of the order of the wavelength of the light In integrated optics, interferometric optical sensors and electro-optic amplitude modulators are often of the Mach-Zehnder interferometer (MZI) type (see $F_{1} g$ 8) In this kind of interferometer a power-splitting $\mathrm{Y}$-junction at the entrance of the 


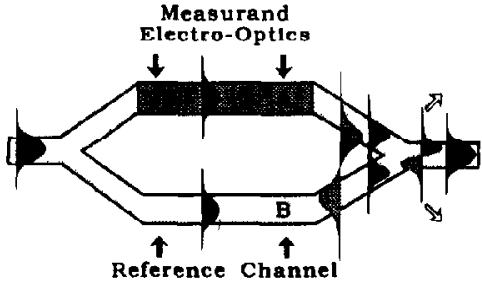

Fig 8 Example of a Mach-Zehnder interferometer

structure is used for dividing the incoming power over two channels During passage through the channels the two beams will undergo phase changes that are dependent on the propagation speed of the beams For optical sensors one beam is normally used as a reference beam (B), whereas the other beam passes through a waveguide (A) in which the propagation constant depends in some way on the value of a quantity that has to be measured (the measurand) [61]

The non-linear counterpart of the MZI (the non-linear Mach-Zehnder interferometer, NMZI) was introduced by Haus et al $[62,63]$ In the NMZI they proposed, the input section of the interferometer has two additional inputs serving for the injection of input signals in both branches Due to non-linearity in the structure, the ac Kerr-ınduced index changes cause the propagatıng modes to gain extra phase changes which are resolved (by interference) as amplitude changes at the output section When using two signal inputs and one gate signal (all signals being pulsed), logical switching ports of the XOR and the AND type can be made

A schematic presentation of the NMZI considered here [48] is given in Fig 9 The input section (left side of Fig 9) of the structure consists of three monomodal waveguides The two outer waveguides are used for injection of signals, which are removed by the two outer waveguides at the

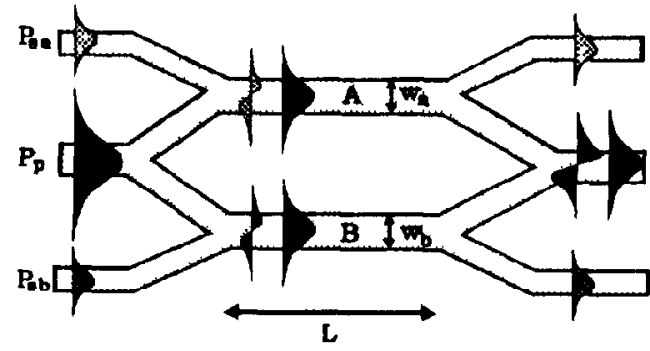

Fig 9 Schematic presentation of the considered NM7.I

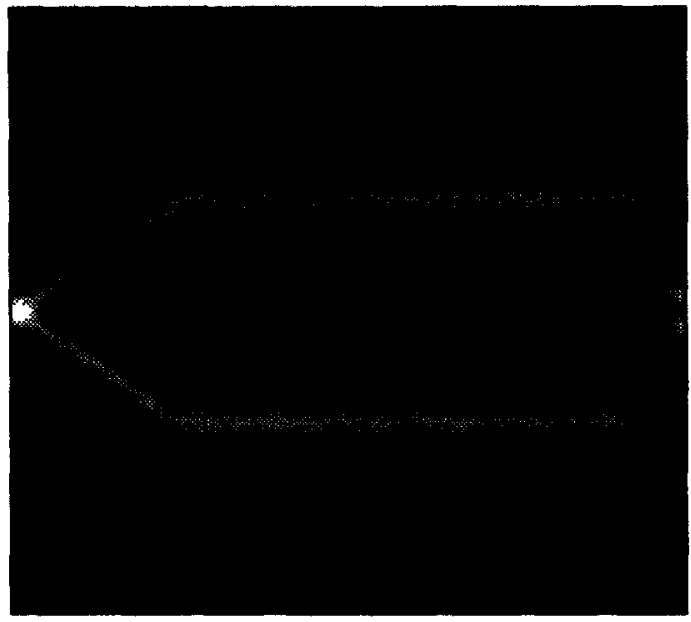

Fig 10 Intensity distribution obtained with BPM simulations on the structure depicted in Fig 9 The intensity of the pump beam is $P_{\mathrm{p}}=1 \mathrm{~kW} / \mathrm{m}$ and that of the signal beam $P_{\mathrm{s}}=56 \mathrm{~W} / \mathrm{m}$, corresponding to the situation that predommantly a first-order mode is excited at the output

output section The middle waveguide is provided with a strong beam, the pump beam The two branches of the NMZI and the central output guide are bimodal The two signal beams affect the phase difference at the central output port, and so the intensity distribution of the two output modes If we isolate the fundamental mode at the output port (for example), the device may serve as an all-optical $(\log 1 \mathrm{c})$ switch or a differential amplifier In Figs 10 and $11 \mathrm{BPM}$ simulations are shown of the NMZI, showing the switching from a $\mathrm{TE}_{1}$

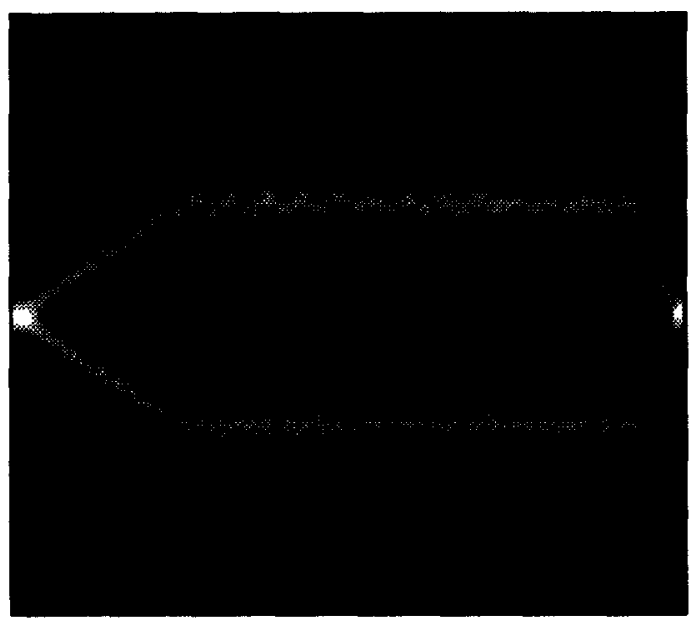

Fig 11 As for Fig 10 , but $P_{\mathrm{s}}=112 \mathrm{~W} / \mathrm{m}$, corresponding to a fundamental mode leaving the output 
output mode to a $\mathrm{TE}_{0}$ output mode, induced by changes in the intensity of the signal beam

We remark that the performance of the NMZI, described above, does not depend, in first approximation, on the intensity of the pump beam [48] This is of importance for unwanted pulse breakup, which often occurs in all-optical switching devices due to the intensity distribution over the pulse

\section{Concluding remarks}

In the past few years a large variety of all-opt1cal devices have been proposed, some of which have been realized and tested To increase applications of such devices, there is still a need for strongly non-linear fast reactıng and (possibly) non-absorbing materials In this field further research, both experimental and theoretical, is desirable in order to obtain more insight into the underlyıng mechanısms and might be of help for the tailoring of materials

Simulations of all-optical devices mostly assume an ideal Kerr-law non-linearity and a $c$ w input However, the response of a device to fast pulses might differ considerably from the predictions obtained by simulations, and pulse break-up may occur The latter effect may possibly be overcome by using temporal solitons [3] Further simulations are nccded, using realıstic material parameters, in order to realize devices with good performance

\section{References}

1 Y R Shen, The Princules of Nonlmear Opttcs, Wiley, New York. 1984

2 F A Hopf and G I Stegeman, Appled Classical Electrodynamics, Vols I and II, Wiley, New York, 1986

3 G I Stegeman and E M Wright, All-optıcal waveguide switching, Opt Quantum Electron, 22 (1990) 95-122

4 R S Potember, R C Hoffman, K A Stetyick and R A Murphy, in A J Heeger, J Orenstein and D R Ulrich (eds), Nonimear Optical Properties of Polymers, Matertals Research Society, Vol 109, 1988, pp 29-34

5 K C Rustagı and J Ducuing, Third-order optical polarizability of conjugated organic molecules, Opt Commun, 10 (1974) 258261

$6 \mathrm{~W}$ Wu and S Kivelson, in A J Heeger, J Orenstein and D R Ulrich (eds), Nonlinear Optical Properties of Polymers, Matenals Research Society, Vol 109, 1988, pp 229-23?

7 C Sauteret, J-P Hermann, R Frey, F Pradere, J Ducuing, R $\mathrm{H}$ Baughman and $\mathrm{R} R$ Chance, Optical nonlinedrities in IDconjugated polymer crystals, Phvs Rev Lett, .36 (1976) 956-959
$8 \mathrm{~W} \mathrm{Wu}$, Nonlinear optical susceptibulities of a one-dimensional semiconductor, Phys Rev Lett, 6l (1988) 1119-1122

9 C Q Wu and X Sun, Third-harmonic generation of polyacetylene, Phvs Rev B, 42 (1990) 9736-9739

$10 \mathrm{X}$ Sun, $\mathrm{K}$ Nasu, C Q Wu and J Liu, Damping and third-order nonlinear spectrum of polyacetylene, paper presented at Mater Res Soc Fall Meeting, Boston, MA, USA, 1991

11 P N Prasad, in P N Prasad and D R Ulrich (eds), Nonlinear Optical and Electroactive Polymers, Plenum New York, 1988, pp $41-67$

12 P Pulay, Derivatives of variational energy expressions $J$ Chem Phrs, 78 (1983) 5043-5051

13 A F Garito, J R Heflın, K Y Wong and O Zamani-Khamırı In A J Heeger, J Orenstein and D $\mathbf{R}$ Ulnch (eds) Nonlmear Optical Properties of Polymers, Materials Research Society Vol 109 1988, pp 91-108

14 W E Torruellas, R Zanoni, G I Stegeman G R Mohlman, E W $P$ Erdhuisen and $W G$ Horsthuis, The cubic susceptibility dispersion of alkoxy-nitro-stilbene (MONS) the di-alkyl-ammonitro-stilbene (DANS) side chain substituted polymers companson with the two level model $J$ Chem Phvs 94 (1991) 68516855

15 A F Garito, Recent progress in nonlınear optical organic and polymer systems and new photonics technologies paper presented at Mater Res Soc Fall Meeting, Boston, MA, USA, 199I

$16 \mathrm{D}$ Yaron and R Sibey The effects of electron correlation on the nonlinear optical properties of polyacetylene paper presented at Mater Res Soc Fall Meeting, Boston MA USA 1991

17 D S Chemla, in C Flytzanıs and J L Oudar (eds), Nonlinear Optics Materials and Devices, Springer, Berlın, 1986, pp 65-78

$18 \mathrm{C} \mathrm{T}$ Seaton, $X$ Mal, G I Stegeman and $H$ G Winful, Nonlinear guided wave applications Opt Eng, 24 (1985) 593-599

$19 \mathrm{M} \mathrm{Kull}$ and J-L Coutaz Intensity-dependent absorption and luminescence in semiconductor-doped glasses, $J$ Opt Soc Am B 7 (1990) 1463-1472

$20 \mathrm{M}$ Tomita and $\mathrm{M}$ Matsuoka, Laser-1nduced irreversible change of the carricr-rccombination process in CdS $\mathrm{Sc}_{1, \text {, }}$-doped glasses, $J$ Opt Soc Am B 7 (1990) 1198-1203

21 M Sinclaır, D McBranch, D Moses and A J Heeger Timeresolved wavegunde modulation of a conjugated polymer $A_{p} p^{\prime}$ Phvs Lett, 53 (1988) 23742377

22 D McBranch, A Hays M Sinclarr D Moses and A J Heeger, Picosecond nondegenerate waveguide modulation in a conjugated polymer, Opt Commun, 81 (1991) 27-32

23 D Ricard, $P$ Roussignol and $C$ Flytzanis Surface-mediated cnhancement of optical phasc conjugation in metal collords, $O p t$ Lett JO (1985) 511-513

24 M Lynch J Hegarty, A Ginty and W M Kelly, paper presented at ECO, The Hague, The Netherlands, March 12-15, 1990

$25 \mathrm{~N}$ L Craft and M E Prise, Processor does light logic Laser Focus World 1990, pp 191-200

26 F Hache, D Ricard and C Flytzanis, Optical nonlinearities of small particles surface-mediated resonance and quantum size effects, $J$ Opt Soc Am, 3 (1986) 1647-1655

$27 \mathrm{~K}$ M Leung, Optical bistability in the scattering and absorption of light from nonlinear nanoparticles Phys $\operatorname{Rev} A, 33$ (1986) $2461-2464$

28 M H Birnboim, J W Haus N Kalyaniwalla W Ping Ma and R Inguva, Structured nanoparticle composites with enhanced intrinsic optical bistability, paper presented at $E C O$ The Hague The Netherlands, March 12-15, 1990

$29 \mathrm{G}$ I Stegeman, E M Wright N Finlayson, R Zanonı and C T Seaton Third order nonlincar integrated optics $J$ Lightwave Technol 6(1988) 953-970

$30 \mathrm{R}$ Ulrich, Theory of prism-film coupler by plane wave analysis, $J$ Opt Soc Am 60 (1970) 1337-1350 
31 R Ulrich, Efficiency of optical gratıng couplers, $J$ Opt Soc Am, 63 (1973) 1419-1431

$32 \mathrm{P} \quad \mathrm{K}$ Tien and $\mathrm{R}$ Ulrich, Theory of prism-film coupler and thin-film light guides, $J$ Opt Soc $A m, 60$ (1970) 1325-1337

33 We believe that the ray picture, as used by [32], should also inlcude the effect of the Goos-Hanchen shift [34] A discussion on this will be published elsewhere

34 H Kogelnik, in T Tamir (ed), Topics in Applued Physics, Springer, Berlın, 1979, pp 13-81

35 G M Carter and Y J Chen, Nonlinear coupling between radiation and confined modes, Appl Phys Lett, 42 (1983) 643-645

36 C Liao and G I Stegeman, Nonlinear prism coupler, Appl Phys Lett , 44 (1984) 164-166

37 B J Offrein, H J W M Hoekstra, T H Hoekstra, A Dressen and Th J A Popma, Coherent detection of the AC-Kerr effect by pump-probe prism coupling in semı-conductor glasses, Internal Report, to be published

38 H M M Klen Koerkamp, $T$ H Hoekstra, A Dnessen, Th J A Popma, G R Mohlman, W H G Horsthus and E W P Erdhuisen, in J A Emerson and J M Torkelson (eds), Opncal and Electrical Properties of Polymers, Materials Research Society, Vol 214, 1991, pp 41-46

39 M Dumont and Y Levy, in T Kobayashı (ed ), Nonlenear Optics of Organics and Semiconductors, Proc Int Symp Tokyo, Japan, 1988, pp 256-266

$40 \mathrm{~W}$ Burns and A Milton, Mode conversion in planar dielectric separatıng waveguides, IEEE $J$ Quantum Electron, $Q E-1 /(1975)$ 32-39

41 G J M Knjnen, H J W M Hoekstra P V Lambeck and Th J A Popma, in E Van Groessen and E $M$ de Jager (eds), Studies in Mathematical Physics, Vol 3, Elsevier, Amsterdam, 1991, pp 481-490

$42 \mathrm{~T}-\mathrm{T}$ Sh 1 and $\mathrm{S} C h 1$, Nonlinear wave propagation in an dsymmetnic converging Y-junction, Opt Lett, 14 (1991) 1077-1079

$43 \mathrm{Y}$ Silberberg and B G Sfez, All-optical phase- and powercontrolled switching in nonlinear waveguide junctions, $O p t$ Lett, 13 (1988) 1132-1134

$44 \mathrm{~K}$ Ogusu, Self-switching in hollow waveguides with a Kerr-like nonlinear permittivity, $J$ Lightwave Technol, 8 (1990) 1541-1547

45 S D Smith, An introduction to optically bistable devices and photonic logic, Philos Trans $R$ Soc London A, $3 / 3$ (1984) 195-204

46 M Haelterman, All-optical set-reset flip-flop operation in the nonlinear Fabry-Perot interferometer, Opt Commun, 86 (1991) $189-191$

47 A Empsten and I Veretennicoff, Optical bistability in a NLDFB device, IEEE $J$ Quantum Electron, QE-26 (1990) 1089-1097

48 G J M Krijnen, Ph D Thesis, University of Twente, 1992

49 R De La Fuente, A Barthelemy and C Froehly, Spatial soliton induced guided waves in a homogeneous nonlincar Kerr medium, Opt Lett, 16 (1991) 793-795

$50 \mathrm{~J}$ S Aitchison, A M Weiner, Y Silberberg, D E Leaird, M K Ohiver, J L Jackel and P W E Smith, Experimental observation of spatial soliton interactions, Opt Lett, 16 (1991) 15-17

51 F B McCormick, A L Lentıne, $R$ L Morrison, S L Walker, L M F Chirovsky and L A D'Asaro, Parallel operation of a $32 \times 16$ symmetric self-electrooptic effect device array, IEEE Photon Technol Lett, 3 (1991) 232-234

$52 \mathrm{~S}$ Fukushima and $\mathrm{T}$ Kurokawa, Optical parallel processor for binary images with cascaded brpolar-operational spatial light modulators, IEEE Photon Technol Lett, 3 (1991) 682684

53 S Noda T Takayama, K Shıbata, Y Kobayashı and A Sasakı, Optical tristable device by vertical and direct integration of hetcrojunction phototransistor and laser diode, Proc Ecoc, Parts, France, 1991 pp $457-460$
$54 \mathrm{M}$ N Islam, C E Soccolich and D A B Miller, Low energy ultrafast fiber soliton logic gates, Opt Lett, 15 (1990) 909_ 911

$55 \mathrm{M}$ N Islam and $\mathbf{J}$ R Sauer, Generalized exclusive-or modules as a natural basis for all-optical fiber logic systems, IEEE $J$ Quantum Electron, $Q E-27$ (1991) 843-848

56 J van Roey, J van der Donk and P E Lagasse, Beam-propagaLoon method analysis and assessment, $J$ Opt Soc Am, 71 (1981) $803-810$

$57 \mathrm{~L}$ Thylen The beam propagation method an analysis of its applicability, Opt Quant Elect, 14 (1983) 433439

$58 \mathrm{D}$ Yevick and B Hermansson, Efficient beam propagation techniques, IEEE J Quantum Electron, $Q E-26$ (1990) 109-112

59 H J W M Hoekstra, G J M Krijnen and P V Lambeck, Efficient interface conditıons for the finite difference beam propagation method, $J$ Lightwave Technol, submitted for publication

$60 \mathrm{H}$ Berends, The properties of nonlmear asymmetrical Y-junctıons, simulational results, Internal Report, University of Twente, 1991

61 H J M Kreuwel, Planar waveguide sensor for the chemical domain, $P h D$ Thesis, University of Twente, 1988

62 A Lattes, H A Haus, $F$ Leonberger and $E$ P Ippen, An ultrafast all-optical gate, IEEE $J$ Quantum Electron, $Q E-19$ (1983) $1718-1723$

$63 \mathrm{H} \mathrm{A}$ Haus and N A Whitaker Jr, All-optical logic in optical waveguides, Phlos Trans $R$ Soc London A, 313 (1984) 310319

\section{Biographies}

Hugo $J W M$ Hoekstra (1949) recelved the M Sc degree in experimental physics from the Unıversity of Amsterdam, The Netherlands, in 1977 After being a teacher in high school, he began Ph D study on magnetooptical properties of solid transition-metal halides at the University of Groningen, where he received the $\mathrm{Ph} D$ degree in 1984

Between 1984 and 1988 he was a postdoctoral fellow in the field of surface science at the Universities of Nijmegen and Groningen In 1988 he joined the Lightwave Device Group of the MESA Research Institute of the University of Twente, where he is engaged in work on linear and nonlinear optics

Gijs J M Krynen was born in The Netherlands in 1961 His M Sc thesis (1987) concerned magnetic recording, an investigation carried out at Philips Laboratories in Eindhoven In 1987 he started his Ph D research in the Lightwave Device Group of the MESA Research Institute of the University of Twente His current $\mathrm{Ph} \mathrm{D}$ work is on the design and realization of all-optical switching devices, and will be finıshed in the early spring of 1992 
Alfred Driessen (1949) recelved a degree in physics in 1972, and in 1982 the Ph D degree, both from the University of Amsterdam

From 1982 to 1987 he worked at the Physics Department of the Free University of Amsterdam on hydrogen in metals and high-temperature superconductors Since 1988 he has been an associate professor in the Lightwave Device Group of the MESA Research Institute of the Unversity of Twente His current research is mainly non-linear optical materials and integrated optics for telecommunication

Paul V Lambeck (1939) recelved the M Sc degree in physical chemistry from the University of Amsterdam in 1964 In the same year he joined the University of Twente, starting in the field of ferroelectric materials, a study which resulted in a $\mathrm{Ph} \mathrm{D}$ thesis
Since 1984 he has been an associate professor in the Lightwave Device Group of the MESA Research Institute of the University of Twente His current interest is in the field of integrated optics and concentrates mainly on optical sensors and non-linear devices

Theo J A Popma was born in Heerenveen, The Netherlands, in 1941 He recelved the $\mathrm{M} \mathrm{Sc}$ and $\mathrm{Ph} \mathrm{D}$ degrees from the University of Groningen in 1966 and 1970, respectively During 1970 and 1971 he was at the IBM Thomas J Watson Research Center in Yorktown Heights (NY)

In 1971 he jomed the Philps Research Laboratories in Eindhoven, where he was employed untıl 1984 In 1983 he was appointed professor of Materials Science in the Departments of Applied Physics and Electrical Engineering of the University of Twente, The Netherlands 